(2)

REVIEW

\title{
New developments in the management of severe skin and deep skin structure infections - focus on tedizolid
}

This article was published in the following Dove Press journal:

Therapeutics and Clinical Risk Management

22 May 2015

Number of times this article has been viewed

\author{
Michael J Durkin' \\ G Ralph Corey ${ }^{2,3}$ \\ 'Department of Medicine, Division \\ of Infectious Diseases, Duke \\ University, Durham, NC, USA; \\ ${ }^{2}$ Department of Medicine, Duke \\ Clinical Research Institute, Durham, \\ NC, USA; ${ }^{3}$ Duke Global Health \\ Institute, Durham, NC, USA
}

\begin{abstract}
Tedizolid, a novel oxazolidinone, is approved for treatment of acute bacterial skin and skin structure infections (ABSSSIs). Tedizolid offers several potential advantages over current ABSSSI treatment options. First, tedizolid has a prolonged half-life, which allows for once-daily dosing. Second, tedizolid has broad spectrum activity against Gram-positive organisms including methicillin-resistant Staphylococcus aureus, coagulase-negative staphylococci, and enterococci. Third, tedizolid, available in both intravenous and oral formulations, has high oral bioavailability, allowing for easy oral step-down therapy. Fourth, in patients who have been prescribed selective serotonin reuptake inhibitors or monoamine oxidase inhibitors, tedizolid may have fewer drug interactions than linezolid. Finally, tedizolid may have fewer or comparatively delayed onset side effects than linezolid, including thrombocytopenia and nausea. This review covers the microbiology, pharmacology, mode of action, and pharmacokinetics of tedizolid as well as patient-focused perspectives such as quality of life, patient satisfaction/acceptability, adherence, and uptake and provides expert opinion on the current use of tedizolid for ABSSSIs and potential future therapeutic applications.
\end{abstract}

Keywords: cellulitis, new antibiotics, oxazolidinones, infectious diseases, MRSA

\section{Introduction to the epidemiology and natural history of severe skin structure infections}

Acute bacterial skin and skin structure infections (ABSSSIs), which include cellulitis/ erysipelas, wound infections, and abscesses, are among the most common infections in health care and pose a tremendous burden on the health care system. ${ }^{1}$ Such infections account for 6.3 million physician office visits and over 850,000 hospital admissions annually in the United States.,3 More importantly, the incidence of ABSSSI appears to be increasing. Between 2000 and 2004, the number of total hospital admissions for ABSSSIs increased by almost 30\%; ${ }^{3}$ between 2005 and 2011, the number of total hospital admissions for ABSSSIs increased by over $17 \% .{ }^{4}$ Similarly, the number of emergency department visits for skin and soft tissue infections has almost tripled from 1.2 million to 3.4 million patients between 1993 and $2005 .^{5}$

A variety of pathogens cause severe skin structure infections. However, Grampositive organisms predominate. In fact, the new treatment algorithm for skin and soft tissue infections, used by the Infectious Diseases Society of America, focuses on antimicrobials against Streptococcus spp. and Staphylococcus aureus, and particularly focuses on methicillin-resistant $S$. aureus (MRSA). ${ }^{6}$ Unfortunately, many treatment options for the outpatient treatment of MRSA infections, including vancomycin, daptomycin, ceftaroline, and telavancin, are only available in intravenous formulations. 
Other oral options have serious drawbacks. For example, clindamycin requires administration three to four times per day. In addition, clindamycin resistance among MRSA isolates appears to be increasing. ${ }^{7,8}$ Although quite active against MRSA, the efficacy of trimethoprim-sulfamethoxazole against Streptococcus pyogenes remains subject to debate. ${ }^{9,10}$ Thus newer, oral treatment options for skin structure infections are needed.

\section{Outline of current and emerging developments in the management of skin structure infections}

A number of new antibiotics have shown efficacy in the treatment of complicated skin and skin structure infections including daptomycin, ${ }^{11}$ ceftaroline, ${ }^{12}$ linezolid, ${ }^{13}$ dalbavancin, ${ }^{14}$ ortiavancin, ${ }^{15,16}$ telavancin, ${ }^{17}$ and tedizolid. ${ }^{18}$ The most recently approved antibiotics fall into two separate classes: lipoglycopeptides (oritavancin and dalbavancin) and oxazolidinones (linezolid and tedizolid).

Lipoglycopeptides have several advantages compared to conventional antibiotic therapy for ABSSSIs. First, both dalbavancin and oritavancin have activity against MRSA, in addition to a broad variety of other Gram-positive pathogens. ${ }^{14,16}$ Second, both agents, which are non-inferior to vancomycin in large double-blind studies, can be conveniently dosed once-weekly. ${ }^{14,16}$ In addition, at least one lipoglycopeptide, dalbavancin, had fewer adverse events compared to standard therapy of vancomycin and linezolid in a randomized controlled trial. ${ }^{14}$ Thus, lipoglycopeptides are more convenient and may be safer.

However, lipoglycopeptides have limitations. First, they are currently limited to intravenous formulations for the treatment of ABSSSIs. Second, they have a long plasma half-life ${ }^{14}$ and cannot be removed via dialysis, ${ }^{19,20}$ thus management of patients with an acute allergic reaction would be very limited. Finally, dalbavancin requires an inconvenient second infusion for the treatment of ABSSSI. ${ }^{14}$

\section{Review of microbiology, pharmacology, mode of action, and pharmacokinetics of tedizolid}

Tedizolid and linezolid are both oxazolidinones and share many characteristics. First, both agents have the same spectrum of activity. Specifically, oxazolidinones are active against Gram-positive bacteria, including multidrug resistant organisms such as MRSA and vancomycin-resistant Enterococci. ${ }^{21}$ Second, both agents share the same mechanism of action: binding to the highly conserved, peptidyl transferase A-site of ribosomal ribonucleic acid (rRNA), thus inhibiting protein synthesis. ${ }^{22}$ Third, both agents have high oral bioavailability ( $86 \%-100 \%)$, allowing for oral step-down therapy. ${ }^{23-25}$ Fourth, the efficacy of both agents is not dependent upon the peak concentration of drug (like aminoglycosides) or the time bacteria are exposed above the minimal inhibitory concentration (like beta-lactams). Rather, the ratio of the free area under the 24-hour concentration-time curve to the minimum inhibitory concentration appears to best predict the bactericidal effect of oxazolidinones. ${ }^{26,27}$

However, tedizolid may have several theoretical advantages over linezolid from a microbiologic standpoint. First, tedizolid has at least four fold greater in vitro activity than linezolid. ${ }^{21,28}$ This increased potency may be related to two reasons: tedizolid accumulates at higher intracellular concentrations in phagocytic cells than linezolid; ${ }^{29}$ and unlike linezolid, tedizolid can bind to additional target sites interactions within the $23 \mathrm{~s}$ rRNA. ${ }^{30}$ Second, due to these additional binding sites, tedizolid retains activity against MRSA strains carrying the linezolid resistance cfr rRNA gene mutation. ${ }^{30,31}$ Therefore, tedizolid has a higher theoretical barrier to resistance than linezolid. In one study, only $16(0.19 \%)$ of 6,884 Gram-positive clinical bacterial isolates demonstrated intermediate or resistant phenotypes to tedizolid. ${ }^{32}$ However, in vitro studies evaluating intracellular antibiotic accumulation, antibiotic potency activity in microbiology laboratories, and location of rRNA binding sites using crystallography and mathematical simulations should not be used as surrogates for clinically relevant outcomes. Further studies, in patients, are required to determine if these theoretical benefits will actually be relevant for patient care.

Tedizolid has one concrete advantage over linezolid from a pharmacology standpoint. Tedizolid half-life values are approximately two fold greater than linezolid. ${ }^{25}$ This allows for once-daily dosing as opposed to twice-daily therapy with linezolid. In addition, increased potency may account for tedizolid's efficacy after only 6 days of therapy (linezolid was given for 10 days). However, the demonstration of shortened therapy may simply reveal that the standard treatment of ABSSSIs by linezolid may well be too long.

Tedizolid also has several theoretical advantages over linezolid from a pharmacology standpoint; however, these findings have yet to be substantially established in humans. First, tedizolid is less likely to inhibit monoamine oxidases than linezolid when evaluated in a laboratory setting. ${ }^{33}$ Human studies have supported these findings, but involved few patients. Second, the risk of serotonin syndrome in mice that were administered tedizolid was not only significantly 
lower than the linezolid group but was also comparable to the placebo group - even with supratherapeutic dosing. ${ }^{33}$ However, further experience with humans on tedizolid and selective serotonin reuptake inhibitors is required. Finally, tedizolid may have less mitochondrial toxicity than linezolid. Although tedizolid inhibits mitochondrial protein synthesis more potently than linezolid, pharmacokinetic data suggest that the duration of inhibition is shorter for tedizolid allowing for mitochondrial recovery - dramatically reducing the potential for myelosuppression and neuropathy in animal models. ${ }^{34}$ Importantly, these findings have yet to be confirmed in clinical studies.

In addition to understanding the similarities and differences between tedizolid and linezolid, health care providers should understand the following background pharmacology information prior to caring for patients on tedizolid. First, tedizolid dose adjustment is not necessary in patients with renal or hepatic impairment ${ }^{35}$ as tedizolid excretion is predominately through the fecal $(80 \%-90 \%)$ route. ${ }^{36}$ Second, pharmacokinetic studies also demonstrated that tedizolid phosphate can be administered without regard to meal status. $^{25}$ Third, as discussed above, tedizolid intravenous and oral pharmacokinetic profiles were similar - making tedizolid an excellent option for oral step-down therapy. ${ }^{25}$ Finally, tedizolid has good penetration into skin and soft tissue - making it an ideal option for ABSSSI. ${ }^{37}$ However, tedizolid penetration in other tissues is currently not wellestablished (although excellent lung penetration in healthy volunteers appears promising).$^{38}$ Thus, prescribers should be cautious about using tedizolid in other conditions.

\section{Patient-focused perspectives such as quality of life, patient satisfaction/ acceptability, adherence, and uptake}

The ESTABLISH-1 and ESTABLISH-2 trials are the largest patent-focused studies of tedizolid to date. Each trial, which will be discussed in detail below, was designed as a noninferiority study comparing tedizolid to linezolid.

The ESTABLISH-1 trial randomized 666 patients with ABSSSIs to oral tedizolid once daily for 6 days versus oral linezolid twice daily for 10 days. ${ }^{39}$ The well-performed, noninferiority, double-blinded study included 81 study centers throughout North America, Latin America, and Europe and was evaluated using a rigorous intent-to-treat analysis and two separate response rates: early clinical treatment response (48-72 hours) and sustained clinical treatment response (11 days). Both linezolid and tedizolid had similar rates of clinical cure for ABSSSIs. The early treatment response rate was $80 \%$ in the tedizolid arm compared to $79 \%$ in the linezolid treatment group. The late treatment response rate was $69 \%$ in the tedizolid phosphate group compared to $72 \%$ in the linezolid group. Treatment-emergent adverse events between the two groups were also similar; however, nausea was statistically less likely in the tedizolid arm than the linezolid arm (9\% vs 13\%).

The ESTABLISH-2 trial randomized 666 patients to intravenous tedizolid once daily for 6 days versus intravenous linezolid twice daily for 10 days, with an option for oral step-down therapy. This non-inferiority, double-blinded study was also well-performed and included 58 study centers throughout North America, Europe, Africa, and Asia and was evaluated using a rigorous intent-to-treat analysis and two separate response rates: early clinical treatment response (48-72 hours) and sustained clinical treatment response (11 days). Both linezolid and tedizolid had similar rates of clinical cure for ABSSSIs. The early treatment response rate was $85 \%$ in the tedizolid arm compared to $83 \%$ in the linezolid treatment group. The late treatment response rate was $87 \%$ in the tedizolid phosphate group compared to $88 \%$ in the linezolid group. The overall rates of treatment emergent adverse events in ESTABLISH-2 trial were similar between the two arms. However, the tedizolid arm had a lower rate of gastrointestinal disorders (diarrhea, nausea, and vomiting) compared to the linezolid arm (16\% vs $20 \%)$. ${ }^{18}$

A Phase two dose-ranging study also demonstrated that tedizolid was well tolerated at all studied doses, with no patients discontinuing treatment because of an adverse event. ${ }^{40}$ Common drug-related adverse events included: nausea $(16.5 \%)$, diarrhea $(8.5 \%)$, vomiting $(6.9 \%)$, and headache $(6.4 \%)$ - all of which were non-dose dependent.

When pooled data from human trials were analyzed, tedizolid was less likely to cause thrombocytopenia than linezolid. ${ }^{27}$ However, the statistically significant decreases were observed beyond 7 days of therapy, after tedizolid had been discontinued. Studies evaluating longer duration tedizolid therapy are lacking. One very small 21 -day Phase 1 study in healthy volunteers suggested that tedizolid may show less thrombocytopenia than linezolid. ${ }^{41}$ Clearly, further data are required to determine if the delayed onset thrombocytopenia observed in linezolid is directly related to higher bone marrow toxicity of linezolid compared to tedizolid or if the delayed onset thrombocytopenia is simply related to a longer duration of therapy. Similarly, larger study populations, likely taking tedizolid for longer durations, are required to evaluate if the lower observed neurotoxicity and drug interactions will also be observed in humans. 


\section{Conclusions, place in therapy}

Tedizolid, in our opinion, is a promising antibiotic for the treatment of ABSSSIs. Indeed, once daily dosing and shorter duration of therapy compared to linezolid and other traditional antibiotics are attractive to prescribers. Tedizolid also has several theoretical benefits over linezolid as well: it may be less likely to cause serotonin syndrome; it may be less likely to interact with monoamine oxidase inhibitors; it may be less prone to developing resistance; it may be more potent than linezolid; and it may be less likely to cause neuropathy.

However, tedizolid has one very important drawback: it is very expensive. The current wholesale price for a 6-day course of tedizolid is $\$ 1,692$ for intravenous therapy and $\$ 2,212$ for oral therapy. ${ }^{42}$ One must query if the advantages of tedizolid are worth the added expense in a condition that has a long list of viable treatment alternatives. ${ }^{6}$ For example, although a 10-day course of linezolid currently costs $\$ 3,393.28$ without insurance, ${ }^{43}$ the price is expected to drop dramatically after it becomes generic; linezolid's patent is expected to expire soon. ${ }^{44}$ Other, more traditional, antibiotics for ABSSSIs which also have excellent MRSA coverage are still very reasonably priced at major pharmacy retailers. For example, trimethoprim-sulfamethoxazole costs less than $\$ 4.00$ for a 30 -day supply, and is as effective as clindamycin for ABSSSIs; ${ }^{45,46}$ doxycycline costs a little over $\$ 70.00$ for a month-long supply. ${ }^{43}$

We look forward to further data to support animal and laboratory studies which suggest that tedizolid may be a viable alternative for patients with a contraindication for linezolid therapy such as for patients on selective serotonin reuptake inhibitors and monoamine oxidase inhibitors. Also, patients with conditions like multidrug resistant mycobacteria, who require linezolid therapy for months, may theoretically benefit from tedizolid.

We also look forward to future studies exploring tedizolid, alone or in combination with other antibiotics, to treat more serious infections such as $S$. aureus bacteremia, endocarditis, prosthetic joint infections, or lung infections. If these studies support the use of tedizolid for more serious infections, stepdown oral tedizolid therapy to complete a 4-week or 6-week course of antibiotics, rather than a course of intravenous antibiotics, would be very attractive to both patients and providers.

In our clinical practice, we will likely continue to use more conventional antibiotics to treat most ABSSSIs, such as cephalexin (when the suspicion for MRSA is low), trimethoprim/sulfamethoxazole, doxycycline, clindamycin, vancomycin, daptomycin, and linezolid. However, in circumstances when a patient assistance program or insurance coverage handles most of the outpatient cost, when compliance is a concern, and when shorter duration therapy is needed, tedizolid should be considered.

\section{Disclosure}

MJD reports no conflicts of interest in this work. GRC has been a clinical trial adjudicator for Cubist Pharmaceuticals and Pfizer; a scientific advisor to Achaogen, Cempra, Forest Laboratories/Cerexa, Rib-X, The Medicines Company, Theravance, and Trius Therapeutics (a subsidiary of Cubist Pharmaceuticals); a consultant to Achaogen, Cempra, Contrafect, Cubist Pharmaceuticals, Forest Laboratories/Cerexa, Furiex, GlaxoSmithKline, Rib-X, The Medicines Company, and Theravance; and a principal investigator for Cempra, Forest Laboratories/Cerexa, GlaxoSmithKline, The Medicines Company, and Theravance.

\section{References}

1. McCaig LF, McDonald LC, Mandal S, Jernigan DB. Staphylococcus aureus-associated skin and soft tissue infections in ambulatory care. Emerg Infect Dis. 2006;12(11):1715-1723.

2. Pallin DJ, Egan DJ, Pelletier AJ, Espinola JA, Hooper DC, Camargo CA Jr. Increased US emergency department visits for skin and soft tissue infections, and changes in antibiotic choices, during the emergence of community-associated methicillin-resistant Staphylococcus aureus. Ann Emerg Med. 2008;51(3):291-298.

3. Edelsberg J, Taneja C, Zervos M, et al. Trends in US hospital admissions for skin and soft tissue infections. Emerg Infect Dis. 2009; 15(9):1516-1518.

4. Kachatryan A PD, Johnson K, et al. Rising US hospital admissions for Gram+ acute bacterial skin and skin structure infections (ABSSSIs). Poster presented at SHM; March 24-27; 2014; Las Vegas, Nevada. Available from: http://content.stockpr.com/duratatherapeutics/db/ Publications/2134/file/3-13c_SHM_HCUP_Poster.pdf. Accessed December 10, 2014

5. Pallin DJ, Espinola JA, Leung DY, Hooper DC, Camargo CA Jr. Epidemiology of dermatitis and skin infections in United States physicians' offices, 1993-2005. Clin Infect Dis. 2009;49(6):901-907.

6. Stevens DL, Bisno AL, Chambers HF, et al. Practice guidelines for the diagnosis and management of skin and soft tissue infections: 2014 update by the Infectious Diseases Society of America. Clin Infect Dis. 2014;59(2):e10-e52.

7. Braun L, Craft D, Williams R, Tuamokumo F, Ottolini M. Increasing clindamycin resistance among methicillin-resistant Staphylococcus aureus in 57 northeast United States military treatment facilities. Pediatr Infect Dis J. 2005;24(7):622-626.

8. Hulten KG, Kaplan SL, Gonzalez BE, et al. Three-year surveillance of community onset health care-associated staphylococcus aureus infections in children. Pediatr Infect Dis J. 2006;25(4):349-353.

9. Bowen AC, Lilliebridge RA, Tong SY, et al. Is Streptococcus pyogenes resistant or susceptible to trimethoprim-sulfamethoxazole? J Clin Microbiol. 2012;50(12):4067-4072.

10. Bergmann R, van der Linden M, Chhatwal GS, Nitsche-Schmitz DP. Factors that cause trimethoprim resistance in Streptococcus pyogenes. Antimicrob Agents Chemother. 2014;58(4):2281-2288.

11. Arbeit RD, Maki D, Tally FP, et al. The safety and efficacy of daptomycin for the treatment of complicated skin and skin-structure infections. Clin Infect Dis. 2004;38(12):1673-1681. 
12. Talbot GH, Thye D, Das A, Ge Y. Phase 2 study of ceftaroline versus standard therapy in treatment of complicated skin and skin structure infections. Antimicrob Agents Chemother. 2007;51(10):3612-3616.

13. Wilcox MH, Tack KJ, Bouza E, et al. Complicated skin and skinstructure infections and catheter-related bloodstream infections: noninferiority of linezolid in a phase 3 study. Clin Infect Dis. 2009;48(2):203-212.

14. Boucher HW, Wilcox M, Talbot GH, Puttagunta S, Das AF, Dunne MW. Once-weekly dalbavancin versus daily conventional therapy for skin infection. N Engl J Med. 2014;370(23):2169-2179.

15. Corey GR, Kabler H, Mehra P, et al. Single-dose oritavancin in the treatment of acute bacterial skin infections. $N$ Engl J Med. 2014;370(23):2180-2190.

16. Corey GR, Good S, Jiang H, et al. Single-Dose Oritavancin Versus 7-10 Days of Vancomycin in the Treatment of Gram-Positive Acute Bacterial Skin and Skin Structure Infections: The SOLO II Noninferiority Study. Clin Infect Dis. 2015;60(2):254-262.

17. Stryjewski ME, Graham DR, Wilson SE, et al. Telavancin versus vancomycin for the treatment of complicated skin and skin-structure infections caused by gram-positive organisms. Clin Infect Dis. 2008;46(11):1683-1693.

18. Moran GJ, Fang E, Corey GR, Das AF, De Anda C, Prokocimer P. Tedizolid for 6 days versus linezolid for 10 days for acute bacterial skin and skin-structure infections (ESTABLISH-2): a randomised, doubleblind, phase 3, non-inferiority trial. Lancet Infect Dis. 2014;14(8): 696-705.

19. Marbury T, Dowell JA, Seltzer E, Buckwalter M. Pharmacokinetics of dalbavancin in patients with renal or hepatic impairment. $J$ Clin Pharmacol. 2009;49(4):465-476.

20. Kumar A, Mann HJ, Keshtgarpour M, et al. In vitro characterization of oritavancin clearance from human blood by low-flux, high-flux, and continuous renal replacement therapy dialyzers. Int J Artif Organs. 2011;34(11):1067-1074

21. Brown SD, Traczewski MM. Comparative in vitro antimicrobial activities of torezolid (TR-700), the active moiety of a new oxazolidinone, torezolid phosphate (TR-701), determination of tentative disk diffusion interpretive criteria, and quality control ranges. Antimicrob Agents Chemother. 2010;54(5):2063-2069.

22. Saini JS, Homeyer N, Fulle S, Gohlke H. Determinants of the species selectivity of oxazolidinone antibiotics targeting the large ribosomal subunit. Biol Chem. 2013;394(11):1529-1541.

23. Dryden MS. Linezolid pharmacokinetics and pharmacodynamics in clinical treatment. J Antimicrob Chemother. 2011;66 Suppl 4 iv7-iv15.

24. Flanagan S, Passarell J, Lu Q, Fiedler-Kelly J, Ludwig E, Prokocimer P. Tedizolid population pharmacokinetics, exposure response, and target attainment. Antimicrob Agents Chemother. 2014;58(11): 6462-6470.

25. Flanagan SD, Bien PA, Munoz KA, Minassian SL, Prokocimer PG. Pharmacokinetics of tedizolid following oral administration: single and multiple dose, effect of food, and comparison of two solid forms of the prodrug. Pharmacotherapy. 2014;34(3):240-250.

26. Andes D, van Ogtrop ML, Peng J, Craig WA. In vivo pharmacodynamics of a new oxazolidinone (linezolid). Antimicrob Agents Chemother. 2002;46(11):3484-3489.

27. Lodise TP, Fang E, Minassian SL, Prokocimer PG. Platelet Profile in Patients with Acute Bacterial Skin and Skin Structure Infections Receiving Tedizolid or Linezolid: Findings from the Phase 3 ESTABLISH Clinical Trials. Antimicrob Agents Chemother. 2014; 58(12):7198-7204

28. Schaadt R, Sweeney D, Shinabarger D, Zurenko G. In vitro activity of TR-700, the active ingredient of the antibacterial prodrug TR-701, a novel oxazolidinone antibacterial agent. Antimicrob Agents Chemother. 2009;53(8):3236-3239.

29. Drusano GL, Liu W, Kulawy R, Louie A. Impact of granulocytes on the antimicrobial effect of tedizolid in a mouse thigh infection model. Antimicrob Agents Chemother. 2011;55(11):5300-5305.
30. Shaw KJ, Poppe S, Schaadt R, et al. In vitro activity of TR-700, the antibacterial moiety of the prodrug TR-701, against linezolid-resistant strains. Antimicrob Agents Chemother. 2008;52(12):4442-4447.

31. Livermore DM, Mushtaq S, Warner M, Woodford N. Activity of oxazolidinone TR-700 against linezolid-susceptible and -resistant staphylococci and enterococci. J Antimicrob Chemother. 2009;63(4):713-715.

32. Sahm DF, Deane J, Bien PA, et al. Results of the Surveillance of Tedizolid Activity and Resistance Program: in vitro susceptibility of Gram-positive pathogens collected in 2011 and 2012 from the United States and Europe. Diagn Microbiol Infect Dis. 2015;81(2):112-118.

33. Flanagan S, Bartizal K, Minassian SL, Fang E, Prokocimer P. In vitro, in vivo, and clinical studies of tedizolid to assess the potential for peripheral or central monoamine oxidase interactions. Antimicrob Agents Chemother. 2013;57(7):3060-3066.

34. Flanagan S, McKee EE, Das D, et al. Nonclinical and pharmacokinetic assessments to evaluate the potential of tedizolid and linezolid to affect mitochondrial function. Antimicrob Agents Chemother. 2015;59(1):178-185.

35. Flanagan S, Minassian SL, Morris D, et al. Pharmacokinetics of tedizolid in subjects with renal or hepatic impairment. Antimicrob Agents Chemother. 2014;58(11):6471-6476.

36. Ong V, Flanagan S, Fang E, et al. Absorption, distribution, metabolism, and excretion of the novel antibacterial prodrug tedizolid phosphate. Drug Metab Dispos. 2014;42(8):1275-1284.

37. Sahre M, Sabarinath S, Grant M, et al. Skin and soft tissue concentrations of tedizolid (formerly torezolid), a novel oxazolidinone, following a single oral dose in healthy volunteers. Int J Antimicrob Agents. 2012;40(1):51-54

38. Housman ST, Pope JS, Russomanno J, et al. Pulmonary disposition of tedizolid following administration of once-daily oral 200-milligram tedizolid phosphate in healthy adult volunteers. Antimicrob Agents Chemother. 2012;56(5):2627-2634.

39. Prokocimer P, De Anda C, Fang E, Mehra P, Das A. Tedizolid phosphate vs linezolid for treatment of acute bacterial skin and skin structure infections: the ESTABLISH-1 randomized trial. JAMA. 2013;309(6):559-569.

40. Prokocimer P, Bien P, Surber J, et al. Phase 2, randomized, doubleblind, dose-ranging study evaluating the safety, tolerability, population pharmacokinetics, and efficacy of oral torezolid phosphate in patients with complicated skin and skin structure infections. Antimicrob Agents Chemother. 2011;55(2):583-592.

41. Prokocimer P BP, Muñoz KA, Aster R. 2008. Hematological effects of TR-701, linezolid and placebo administered for 21 days in healthy subjects. Poster F1-2069a Abstract presented at: 48th Interscience conference of Antimicrobial Agents and Chemotherapy (ICAAC) and the Infectious Diseases Society of America (IDSA), 46th Annu. Meet., October 25-28, 2008. Washington, DC.

42. Wong E, Rab S. Tedizolid phosphate (sivextro): a second-generation oxazolidinone to treat acute bacterial skin and skin structure infections. P T. 2014;39(8):555-579.

43. costco.com [homepage on the Internet]. Costco Pharmacy Drug Directory. Available from: http://www.costco.com/Pharmacy/drug-directory-main? storeId=10301\&catalogId=10701\&langId=-1. Accessed February 1, 2015.

44. Chase-Ortiz M. The Future Deals in the Generic Industry. Presented at: Drug, Chemical and Associated Technologies Week; March 15-18; 2010; New York City, NY.

45. walmart.com [homepage on the Internet]. Walmart Retail Prescription Program Drug List; Revised December 31; 2013. Available from: http://i.walmart.com/i/if/hmp/fusion/genericdruglist.pdf. Accessed February 3, 2015.

46. Miller LG, Daum RS, Creech CB, et al. Clindamycin versus trimethoprimsulfamethoxazole for uncomplicated skin infections. $N$ Engl J Med. 2015;372(12):1093-1103. 


\section{Publish your work in this journal}

Therapeutics and Clinical Risk Management is an international, peerreviewed journal of clinical therapeutics and risk management, focusing on concise rapid reporting of clinical studies in all therapeutic areas, outcomes, safety, and programs for the effective, safe, and sustained use of medicines. This journal is indexed on PubMed Central, CAS,

EMBase, Scopus and the Elsevier Bibliographic databases. The manuscript management system is completely online and includes a very quick and fair peer-review system, which is all easy to use. Visit http://www.dovepress.com/testimonials.php to read real quotes from published authors.

Submit your manuscript here: http://www.dovepress.com/therapeutics-and-clinical-risk-management-journal 